\title{
How accurate is the neurosurgery literature? A review of references.
}

\author{
Thiago S Montenegro \\ Thomas Jefferson University \\ Kevin Hines \\ Thomas Jefferson University \\ Glenn A Gonzalez \\ Thomas Jefferson University \\ Umma Fatema \\ Thomas Jefferson University \\ Paul P Partyka \\ Rowan University \\ Follow this and additional works at: https://jdc.jefferson.edu/neurosurgeryfp \\ Part of the Neurology Commons, and the Surgery Commons

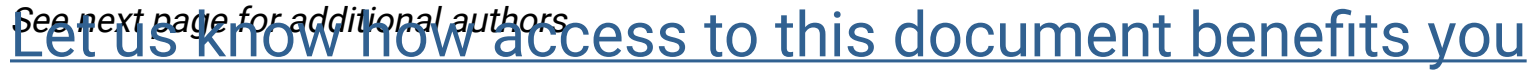

\section{Recommended Citation}

Montenegro, Thiago S; Hines, Kevin; Gonzalez, Glenn A; Fatema, Umma; Partyka, Paul P; Thalheimer, Sara; and Harrop, James, "How accurate is the neurosurgery literature? A review of references." (2020). Department of Neurosurgery Faculty Papers. Paper 139.

https://jdc.jefferson.edu/neurosurgeryfp/139

This Article is brought to you for free and open access by the Jefferson Digital Commons. The Jefferson Digital Commons is a service of Thomas Jefferson University's Center for Teaching and Learning (CTL). The Commons is a showcase for Jefferson books and journals, peer-reviewed scholarly publications, unique historical collections from the University archives, and teaching tools. The Jefferson Digital Commons allows researchers and interested readers anywhere in the world to learn about and keep up to date with Jefferson scholarship. This article has been accepted for inclusion in Department of Neurosurgery Faculty Papers by an authorized administrator of the Jefferson Digital Commons. For more information, please contact: JeffersonDigitalCommons@jefferson.edu. 


\section{Authors}

Thiago S Montenegro, Kevin Hines, Glenn A Gonzalez, Umma Fatema, Paul P Partyka, Sara Thalheimer, and James Harrop 


\title{
How accurate is the Neurosurgery literature? A review of references
}

Thiago S. Montenegro MD ${ }^{1}$, Kevin Hines $\mathrm{MD}^{1}$, Glenn A. Gonzalez MD ${ }^{1}$, Umma Fatema BS ${ }^{1}$, Paul P. Partyka PhD ${ }^{2}$, ${ }^{3}$, Sara Thalheimer BA ${ }^{1}$, James Harrop MD ${ }^{1}$

\author{
${ }^{1}$ Department of Neurosurgery, Thomas Jefferson University and Jefferson Hospital for Neuroscience, Philadelphia, \\ Pennsylvania \\ ${ }^{2}$ School of Osteopathic Medicine, Rowan University, Stratford, New Jersey \\ ${ }^{3}$ Department of Biomedical Engineering, Rowan University, Glassboro, New Jersey \\ Thiago S. Montenegro, MD, Division of Spine and Peripheral Nerve Surgery \\ Department of Neurological Surgery, Thomas Jefferson University Hospital, 901 Walnut Street 3rd Floor, \\ Philadelphia, Pennsylvania 19107. \\ (215) 955-7000 \\ E-mail: thiago.scharthmontenegro@jefferson.edu
}

No portion of the paper has been published, or is under review in any other journal.

Conflict of Interest: None

Disclosure of funding: None

Presentation at a conference: This paper has not been presented in any conference 


\section{Abstract:}

Background: The reference list is an important part of academic manuscripts. The goal of this study is to evaluate the reference accuracy in the field of neurosurgery.

Methods: This study examines four major peer-reviewed neurosurgery journals, chosen based on their clinical impact factor: Neurosurgery, J Neurosurg, World Neurosurg, and Acta Neurochir. For each of the four journals, five articles from each of the journal's 12 issues published in 2019 were randomly selected using an online generator. This resulted in a total of 240 articles; 60 from each journal. Additionally, from each article's list of references, one reference was again randomly selected and checked for a citation or quotation error. The chi-square test was used to analyze the association between the occurrence of citation and quotation errors and the presence of hypothesized risk factors that could impact reference accuracy.

Results: $62.1 \%$ of articles had a minor citation error, $8.33 \%$ had a major citation error, $12.1 \%$ had a minor quotation error, and 5.8\% of articles had a major quotation error.Overall, Acta Neurochir presented with the fewest quotation errors compared to the other journals evaluated. The only association between the frequency of errors and potential markers of reference mistakes was with the length of the bibliography. Surprisingly, this correlation indicated that the articles with longer reference lists had fewer citation errors $(\mathrm{p}<0.01)$. Statistical significance was found between the occurrence of citation errors and the journals of publication $(\mathrm{p}<0.01)$.

Conclusions: In order to advance medical treatment and patient care in neurosurgery, detailed documentation and attention to detail is necessary. The results from this analysis illustrate that improved reference accuracy is required.

Key Words: Reference accuracy, reference list, citation error, quotation error, neurosurgery literature 


\section{Introduction}

The reference list is an important part of academic manuscripts. Authors rely on references to validate and lend authority to the argument being made. Therefore, reference lists serve for writers, researchers, and readers as a vital source on the confirmation of the author's statements accuracy.

The number of articles published in the field of neurosurgery has greatly increased in recent years. [10,17] Because of the rapid pace at which new articles are published, the accuracy of the information circulated is integral to validate research findings. Unfortunately, reference errors in medical literature are common and occur in all specialties.[16,7,12,15,1,4,3,11] In most cases, these errors are trivial, with few impacts in conclusions. However, even small errors can cause misinterpretation and inaccurate data perpetuation and potentially have clinical consequences.[3]

Prior to the present analysis, an equivalent examination was completed of four spine journals. The results were alarming, and demonstrated that improved accuracy to references and quoting references is required.[13] However, to our knowledge, this paper is the first of its kind to evaluate reference errors in the general neurosurgery field, and this was the main motivation that encouraged this study.

Reference errors can be classified as citation or quotation errors, and the incidence of the errors can serve as a measure of reference accuracy in a manuscript.[2] Citation errors consist of inaccuracies in the components of the cited reference that potentially jeopardize the identification of the source. Quotation errors involve inconsistency between the author's assertion and the content of its supporting source. The objective of this study was to quantify the rate of reference errors in neurosurgery literature.

\section{Methods}

Four major peer-reviewed general neurosurgery journals were chosen based on their clinical impact factor: Neurosurgery, J Neurosurg, World Neurosurg, and Acta Neurochir. 2019 journal issues were evaluated. All the articles, from each monthly issue, were numbered sequentially, and 5 articles from each issue were randomly selected using a computer number-generator to determine which articles to include in the study. Articles written for and included in supplements, editorials, letters, and correspondence were excluded due to potential bias in the review process. A sample of 240 articles was selected and analyzed, 60 articles from each journal.

From the reference list of each article selected, all the references were sequentially numbered, and a computer generator was used to randomly select one reference from each article. References from personal communications, letters to the editor, textbook chapters, governmental reports, presentations, and journal articles that were not in English were excluded from the selection. In this case, a further random number was generated, and the resultant reference examined. 
All the articles selected were retrieved and analyzed by four independent investigators. Each referenced article was read completely and thoroughly and compared with the citation and content of the referring article[2] using a standardized checklist to be evaluated for citation and quotation errors. All the citations were checked against information retrieved directly from the official website of each journal analyzed. When a discrepancy was found between reviewers, a fifth senior author reviewed the reference, and the error status was assigned by the consensus of a panel of 5 investigators. Methods were completed in a protocol similar to prior reference study.[13]

Citation errors were categorized as major and minor errors. Major citation errors were those that prevented immediate identification of the source of reference[5]. These included incorrect or omitted publication year, journal title, volume number, supplement designation, or inaccurate pagination not overlapping the correct pages. Minor citation errors included incorrect or omitted issue number, misspellings, omissions, errors of punctuation or substitutions in the authors' names and the title, inaccurate pagination that overlapped the correct pages. $[9,2,11,13]$

Further, quotation errors were classified as major and minor errors. Major quotation errors were determined to occur when the content of the original article failed to substantiate, contradicted, or was unrelated to the authors' contention.[9,4,16,13] A minor quotation error included oversimplification, incorrect measurement numbers, conclusions not reached by the authors of the cited reference and indirect referencing and was noted if the authors' assertion, although inaccurate, preserved the meaning of the original article.[9,4,16,13]

All citation and quotation errors were entered in an excel file; if there were citation and quotation errors in the same reference, both errors were counted separately, but if a reference with a citation error was quoted several times, it was counted just as one error. All quotes related to the same reference were checked, but if there was a minor and a major quotation error, just the major error was counted. In order to calculate the overall error rates, no more than one citation and one quotation error per article were counted. The following items were recorded: the number of authors, the country of origin of the first author, journal of publication, the year of publication of the selected references, and the length of the bibliography for each selected article in order to evaluate if those variables are related to the error rate.[9]

\section{Statistical analysis:}

Data were collected and transferred for analysis to SPSS 26.0 for Windows (Chicago, Illinois, USA). A chi-square test was used to analyze the association of occurrence of citation and quotation errors with the following: journal of publication; the official language of the country of origin of the first author (English vs. non-English); the number of authors ( $\leq 4$ or $>4$ authors); the length of bibliography $(1-15,16-30,31-45,>45$ entries); the year of publication of the reference $(\geq 2014$ or $<2014)$. $[9,13]$ All statistical tests were computed at the $95 \%$ level of confidence.

\section{Results}


A total of 240 articles from the 2019 journal issues were analyzed. Of the total articles verified, $162(67.5 \%)$ had at least one citation or quotation error, 30 (12.5\%) articles had both citation and quotation errors, 10 (4.16\%) had only quotation errors, and 119 (49.58\%) had only citation errors. The total number of articles with citation errors, independent of quotation errors, was 151 (62.9\%), 20 articles (8.33\%) had major citation errors, and 149 (62.1\%) had only minor citation errors. 40 (16.66\%) articles had quotation errors, of which 14 (5.8\%) were major errors and $25(10.41 \%)$ minor errors. (See tables 1 and 2 )

Considering the large proportion of minor citation errors attributed to mistakes on the issue numbers, the percentage of citation errors disregarding it as a mistake was also calculated. In this scenario 84 (35.0\%) articles with at least one citation or quotation error were found, 11 (4.6\%) articles had both citation and quotation errors, 32 (13.3\%) articles had only quotation errors, and $41(17.1 \%)$ had only citation errors (Table 1). From this perspective, the most common type of citation error would be mistakes in punctuation or substitutions in the authors' names (44.82\% of all citation errors counted).

The overall rate of citation error was $62.9 \%$, and $13.2 \%$ of these articles had major errors, while $10.2 \%$ had both major and minor errors in the same article. The most common type of citation error was the incorrect or omitted issue number (70.5\% of all citation errors counted) followed by errors of punctuation or substitutions in the authors' names (13.2\% of all citation errors counted), both considered minor errors in our study. The most common type of major citation error was the inaccuracy of the journal name (48.0\% of all major errors and $6.1 \%$ of all citation errors counted). Thirty-seven citations (15.4\% of all citations) had more than one error, nine citations (3.8\%) had more than two errors, and three citations $(1.3 \%)$ had more than three errors.

The overall quotation error rate was $16.6 \%$, of which $35.0 \%$ were major quotation errors, when the content of the original article failed to substantiate, contradicted, or was unrelated to the authors' contention. (Table 1 and 3 ) All the four journals analyzed had major quotation errors, and its rates ranged from 1.7 to $10 \%$ of all the quotations assessed in its issues. The minor quotation error rate ranged from 6.7 to $20.0 \%$. Figure 1, Tables 2 and 3, summarizes the citation and quotation errors for each journal. The distribution of the mean number of errors (citation and quotation) per article stratified by each journal can be visualized on figure 2 .

The chi-square test was used to analyze the association between the occurrence of citation and quotation errors and the presence of risk factors that could impact reference accuracy. The association between the citation and quotation errors and the following risk factors: number of authors of the articles; the language of the country of origin of the first author; and the year of publication of the reference analyzed was found to be not statistically significant $(p>0.05)$. The association of citation error and the length of the bibliography was statistically significant, but surprisingly showed a greater number of errors in articles with a smaller bibliography (1-15 references), although the quotation error rate was not associated with the bibliography length. (Figure 3) The association between the citation and quotation errors was also not significant. (Table 3) 
When the association between the occurrence of citation errors and the journal of publication was tested, a statistical significance $(\mathrm{p}<0.05)$ was found. However, no statistical significance was found between the association of occurrence of quotation errors and the journal of publication tested. (Table 3)

\section{Discussion}

The process of pursuing accurate references in a journal article may seem simple or trivial, yet citations in today's journals contain a high rate of errors. Authors and readers use references as a source to support and validate research points, and these errors may impede proper substantiation of authors' claims or credit for original research. Even worse than preventing propagation of relevant prior research, quotation errors of prior studies may misrepresent others' research and lead readers to erroneous conclusions that may be propagated continuously in future literature.

Due to the rapid growth rate of neurosurgical literature, proper identification of supporting publications and accurate use is paramount. When examining citation errors in these journals, it appears that neurosurgery literature contains a higher percentage of errors than other specialty literature. However, upon careful examination, the $67.5 \%$ overall error was inflated, as $48.14 \%$ of all these errors were omitted issue number. When examining citation instructions for the journals, it was often not specified that the issue number was required in the citation. In addition, despite the missing issue, the articles were all easily identified despite this error. As a result, the $35.0 \%$ error rate was more reflective of the citation error rate in these journals. This is consistent with many other specialties' literature such as emergency medicine, dentistry, pharmacy, orthopedics, general surgery, spine surgery, otolaryngology, dermatology, and anesthesia that demonstrated error rates ranging approximately from $10 \%$ to $50 \%$.[15,6,14,11,4,7$9,12,13]$ While all of these specialties should strive to standardize and enforce literature citation, most errors in this study were minor, and all articles were easily searched and located in online databases.

The more relevant error prevalent in general neurosurgical literature is quotation error, given the fact that the cited articles were still discoverable. By unintentionally misquoting an article, the meaning of the research is misrepresented and may misinform readers. Once again, this study's error rate was similar to other specialties, and to the spine surgery literature.[15,6,14,11,4,7-9,12,13] With literature error rates of approximately $7 \%$ to $38 \%$ in quoting sources, a significant number of statements may alter the meaning of the cited literature, perpetuating erroneous interpretations of data that may be cited in the future publications.[12]

In this series, citation errors were significantly associated with the journal of publication. Still, there was no significant association with previously mentioned risk factors for citation errors, such as the number of authors, nonEnglish speaking country of origin, or year of publication. However, different than was previously reported and expected, the articles with the lower number of references had a higher number of citation errors. 
The most evident impact of citation errors identified in this study is that the credit given to other's cited original research could have been compromised, given the fact that even minor citation errors could lead to a loss of the citation in an author citation index or a decrease in calculated author's H-index. Perhaps even more importantly, quality research results may be lost and not contribute to current practice. Nevertheless, the quotation errors' impact is even more concerning as it may modify the meaning and perpetuate erroneous interpretations that can affect patient care.

Lastly, while the authors congratulate Acta Neurochir for achieving the lowest number of quotation errors and minimal citation errors, performing well on both fronts, we advocate that all neurosurgery journals work towards a meticulous and rigorous evaluation of both citation and quotation of literature. As the literature for neurosurgery grows, citations and quotations will constitute the foundation needed to efficiently deliver the prior evidence upon which future research will be built.

\section{Conclusion}

Reference inaccuracy is common in the neurosurgery literature occurring in more than a third of the references examined in this study. Medical manuscripts are the mainstay to highlight and propagate advances in medical treatment. Meticulous documentation and attention to detail are necessary in order to advance patient care. This review of the reference accuracy in neurosurgical journals illustrates that improved accuracy to references and quoting references is required. 


\section{Declarations}

Funding: No funding was received for this research.

Conflict of Interest: All authors certify that they have no affiliations with or involvement in any organization or entity with any financial interest (such as honoraria; educational grants; participation in speakers' bureaus; membership, employment, consultancies, stock ownership, or other equity interest; and expert testimony or patent-licensing arrangements), or non-financial interest (such as personal or professional relationships, affiliations, knowledge or beliefs) in the subject matter or materials discussed in this manuscript.

Ethical approval: All procedures performed in studies involving human participants were in accordance with the ethical standards of the institutional and/or national research committee and with the 1964 Helsinki declaration and its later amendments or comparable ethical standards. For this type of study formal consent is not required.

Informed consent: For this type of study informed consent is not required. 


\section{Reference}

1. Al-Benna S, Rajgarhia P, Ahmed S, Sheikh Z (2009) Accuracy of references in burns journals. Burns 35:677-680. doi:10.1016/j.burns.2008.11.014

2. Armstrong MF, Conduff JH, Fenton JE, Coelho DH (2018) Reference Errors in OtolaryngologyHead and Neck Surgery Literature. Otolaryngology-Head and Neck Surgery 159:249-253. doi:10.1177/0194599818772521

3. Awrey J, Inaba K, Barmparas G, Recinos G, Teixeira PG, Chan LS, Talving P, Demetriades D (2011) Reference accuracy in the general surgery literature. World J Surg 35:475-479. doi:10.1007/s00268-010-0912-7

4. Davids JR, Weigl DM, Edmonds JP, Blackhurst DW (2010) Reference accuracy in peerreviewed pediatric orthopaedic literature. JBJS 92:1155-1161. doi:10.2106/JBJS.I.00063

5. De Lacey G, Record C, Wade J (1985) How accurate are quotations and references in medical journals? Br Med J (Clin Res Ed) 291:884-886. doi:10.1136/bmj.291.6499.884

6. Doms CA (1989) A Survey of Reference Accuracy in Five National Dental Journals. J Dent Res 68:442-444. doi:10.1177/00220345890680030101

7. Evans JT, Nadjari HI, Burchell SA (1990) Quotational and reference accuracy in surgical journals. A continuing peer review problem. JAMA 263:1353-1354. doi:10.1001/jama.1990.03440100059009

8. Fenton J, Brazier H, De Souza A, Hughes J, McShane D (2000) The accuracy of citation and quotation in otolaryngology/head and neck surgery journals 1 . Clin Otolaryngol Allied Sci 25:40-44. doi:10.1046/j.1365-2273.2000.00322.x

9. George PM, Robbins K (1994) Reference accuracy in the dermatologic literature. J Am Acad Dermatol 31:61-64. doi:10.1016/s0190-9622(94)70136-9

10. Hauptman JS, Chow DS, Martin NA, Itagaki MW (2011) Research productivity in neurosurgery: trends in globalization, scientific focus, and funding: a review. J Neurosurg 115:1262-1272. doi:10.3171/2011.8.JNS11857

11. Luo M, Li CC, Molina IV D, Andersen CR, Panchbhavi VK (2013) Accuracy of citation and quotation in foot and ankle surgery journals. Foot Ankle Int 34:949-955. doi:10.1177/1071100713475354

12. McLellan MF, Case LD, Barnett MC (1992) Trust, but verify. The accuracy of references in four anesthesia journals. Anesthesiology 77:185-188. doi:10.1097/00000542199207000-00024

13. Montenegro TS, Hines K, Partyka P, Harrop J (in press) Reference Accuracy in Spine Surgery. J Neurosurg Spine

14. Neihouse PF, Priske SC (1989) Quotation accuracy in review articles. DICP 23:594-596. doi:10.1177/1060028089023007-813

15. O'Connor AE (2002) A review of the accuracy of references in the journal Emergency Medicine. Emerg Med 14:139-141. doi:10.1046/j.1442-2026.2002.00307.x

16. Reddy M, Srinivas S, Sabanayagam N, Balasubramanian S (2008) Accuracy of references in general surgical journals - an old problem revisited. The Surgeon 6:71-75. doi:10.1016/s1479-666x(08)80067-4 
17. Wei M, Wang $W$, Zhuang $Y$ (2016) Worldwide research productivity in the field of spine surgery: a 10-year bibliometric analysis. Eur Spine J 25:976-982. doi:10.1007/s00586016-4442-3 


\section{Figure captions}

Figure 1. Number of citation and quotation errors by journal

Figure 2. - Violin Plot for the distribution of the number of errors per article stratified by the journals analyzed. Width of the violin indicates density of cases at a given mean number of errors per article. Solid lines are the mean number of errors per article. Dashed lines represent the interquartile range

Figure 3. Mean number of citation and quotation errors stratified by the length of bibliography 